\title{
Loss of Function of KCNC1 is associated with intellectual disability without seizures
}

\author{
Karine Poirier ${ }^{1,2,3}$, Géraldine Viot $^{4}$, Laura Lombardi ${ }^{1,2,3}$, Clémence Jauny ${ }^{4}$, Pierre Billuart ${ }^{1,2,3}$ \\ and Thierry Bienvenu $u^{\star, 1,2,3,5}$
}

p.(Arg320His) mutation in the $K C N C 1$ gene in human $11 \mathrm{p} 15.1$ has recently been identified in patients with progressive myoclonus epilepsies, a group of rare inherited disorders manifesting with action myoclonus, myoclonic epilepsy, and ataxia. This $K C N C 1$ variant causes a dominant-negative effect. Here we describe three patients from the same family with intellectual disability and dysmorphic features. The three affected individuals carry a c.1015C $>\mathrm{T}$ (p.(Arg339*)) nonsense variant in KCNC1 gene. As previously observed in the mutant mouse carrying a disrupted $K C N C 1$ gene, these findings reveal that individuals with a KCNC1 loss-of-function variant can present intellectual disability without seizure and epilepsy.

European Journal of Human Genetics (2017) 25, 560-564; doi:10.1038/ejhg.2017.3; published online 1 February 2017

\section{INTRODUCTION}

Potassium channels constitute a very diverse group of ion channels and are composed of variable combinations of subunits encoded in large multigene families. ${ }^{1}$ This diversity contributes to the ability of specific neurons to respond uniquely to different inputs. One of these groups of genes, the Kv family, encodes subunits of tetrameric voltagegated $\mathrm{K}^{+}$channels and is divided into several subfamilies based on sequence similarities and hence probable evolutionary relationships. ${ }^{1,2}$ Subunits of the same subfamily, but not from different subfamilies, can form heteromeric channels, suggesting that each group of genes encodes a distinct set of channels. ${ }^{1}$ One of the Kv subfamilies known as $\mathrm{Kv} 3$ has generated particular interest because their members show large unit conductances, high thresholds of activation, and very fast activation and deactivation kinetics. ${ }^{3} \mathrm{Kv} 3$-type channels are involved in the rapid repolarization of the action potential (AP), and their presence in neurons correlates with narrow APs, fast after hyperpolarization (AHP), and high-frequency firing beyond $200 \mathrm{~Hz}$.

The Kv3 subfamily consists of four genes (Kv3.1 (also known as KCNC1), Kv3.2, Kv3.3, and Kv3.4). Each Kv3 gene encodes multiple products by alternative splicing of $3^{\prime}$ ends resulting in the expression of $\mathrm{K}^{+}$channel proteins that differ only in short C-terminal sequences. ${ }^{4}$ The variant $\mathrm{C}$ termini contains or regulates targeting signals and raises the possibility that alternative splicing of $3^{\prime}$ ends of other $\mathrm{K}^{+}$channel genes has similar roles. ${ }^{5,6}$ Although Kv3.1 and Kv3.3 subunits are expressed throughout the brain, their expressions are restricted to distinct neuronal subpopulations. ${ }^{7}$ In neocortex, thalamus, hippocampus, and striatum, Kv3-type channels are found in inhibitory GABAergic interneurons that also express the calcium-binding protein parvalbumin, a marker for fast-spiking neurons. However, $\mathrm{K}_{\mathrm{v}} 3.1$ channels were identified not only in neurons, but also in T-lymphocytes, oligodendrocyte precursor cells, astrocytes, and NPCs. ${ }^{8-10}$

Ten years ago, de novo or autosomal dominantly KCNC3 $\left(\mathrm{K}_{\mathrm{V}} 3.3\right)$ missense variants were identified in spinocerebellar ataxia. ${ }^{11}$ In 2013 ,
Rajakulendran et al ${ }^{12}$ identified a $670 \mathrm{~Kb}$ deletion of chromosome 12q21 including KCNC2 and ATXN7L3B in patients with neurodevelopmental delay and ataxia. More recently, recurrent de novo mutation c.959G > A (p.(Arg320His)) in KCNC1 was identified as a novel major cause for progressive myoclonus epilepsies, a group of rare, inherited disorders manifesting with action myoclonus, tonic-clonic seizures, and ataxia. ${ }^{13}$

During the genetic characterization of a large cohort of patients with syndromic and non-syndromic forms of intellectual disability (ID), we identified a nonsense variant in the KCNC1 gene for three affected individuals of the same family. Affected father, his daughter, and his son suffered from intellectual disability and dysmorphic features without seizures.

\section{SUBJECTS AND METHODS \\ Patients}

We report a family with three affected people: a father, his daughter and his son. All presented with delayed motor milestones, speech delay, hypotonia, lability of attention, intellectual disability, normal head circumference (Figure 1a), minor dysmorphic features included epicanthal folds, ptosis, short philtrum, prognathism, fetal pads, and protruding ears. The grandparents were unaffected. The couple was a pair of cousins but the grandparents were not related.

This family was ascertained in 2011 when individual-2 was referred for genetic counseling, because her husband and daughter have intellectual disability (Figure 1a). The affected girl (individual-3) was born at term after an uneventful pregnancy and delivery. At birth, measurements were normal (weight $(2740 \mathrm{~g})$, height $(51 \mathrm{~cm})$, and head circumference $(34 \mathrm{~cm})$ ). She had mild developmental delay. She began crawling at 11 months and walking at 19 months. She had dysmorphic features including prognathism, cup-shaped ears, protruding tongue, salivary incontinence, and clinodactylie of the fifth finger. At the age of 6 , she had intellectual disability with severe attention and learning difficulties. Neurological examinations appeared to be normal with no ataxia and pyramidal disorder. She did not exhibit any signs of breathing or

${ }^{1}$ Inserm, U1016, Institut Cochin, Paris, France; ${ }^{2} \mathrm{Cnrs}$, UMR8104, Paris, France; ${ }^{3}$ Université Paris Descartes, Sorbonne Paris Cité, Paris, France; ${ }^{4}$ Unité de Génétique Clinique, Maternité Port-Royal, HUPC, Hôpital Cochin, Paris, France; ${ }^{5}$ Assistance Publique-Hôpitaux de Paris, Groupe Universitaire Paris Centre, Site Cochin, Laboratoire de Biochimie et Génétique Moléculaire, Paris, France

${ }^{*}$ Correspondence: Professor T Bienvenu, Université Paris Descartes, Institut Cochin, 24 rue du Faubourg St Jacques, 75014 Paris, France. Tel: +33 144412479 ; Fax: +33 15841 15 80; E-mail: thierry.bienvenu@inserm.fr

Received 3 June 2016; revised 9 December 2016; accepted 24 December 2016; published online 1 February 2017 
a

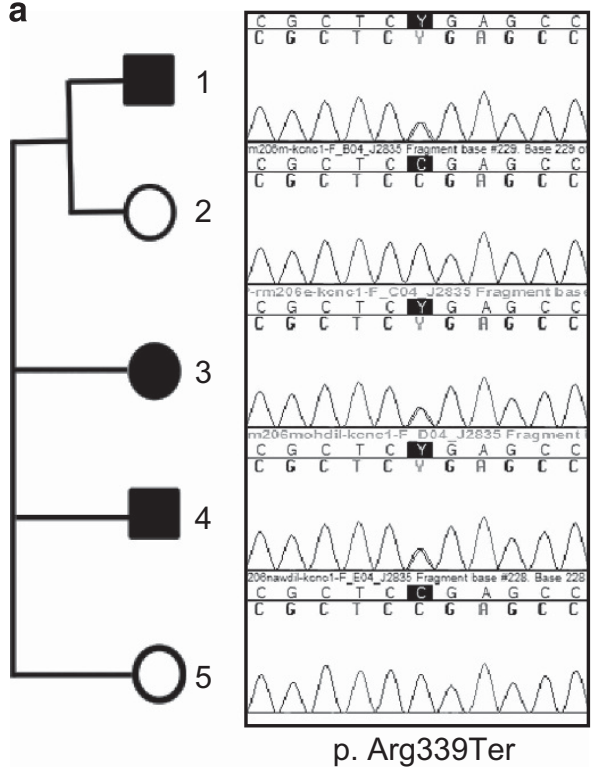

b

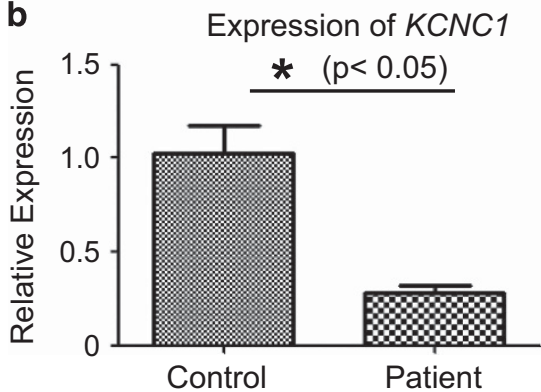

Figure 1 (a) Pedigree of the ID family, Electrophoregram of the $\mathrm{KCNCl}$ nonsense mutation (p.(Arg339Ter)) (indicated by the symbol $Y$ ) identified in one, three, and four family members. (b) Consequence of the nonsense mutation at the mRNA level. Analysis of $K C N C 1$ mRNA extracted from fibroblasts by real-time quantitative reverse-transcriptase PCR. Ctrl: controls $(n=13)$. Patient: 3 .

sleeping anomalies. At the age of 10 , the measurements were within normal ranges (weight $(29 \mathrm{~kg})$, height $(135 \mathrm{~cm} ;+0.9$ DS) and head circumference $(52 \mathrm{~cm}))$. Brain MRI and EEG were normal. The father also presented prognathism, dysplastic ears, and coarse face with moderate intellectual disability. Unlike his brothers and sisters, he was unable to read and write, and developed simple language. He was admitted and worked in a special institution. He is under guardianship to manage his money and administrative procedures. He is now taken care for a brain tumor discovered at the age of 40 after an epileptic seizure. The second boy sibling (individual-4) exhibited intellectual disability with hypotonia and the same face. Any regression and no progressive myoclonus epilepsy were reported for all affected patients.

First, we screened the whole coding regions of 20 previously ID-genes (MECP2, CDKL5, ARX, STXBP1, MEF2C, ARID1B, OPHN1, TBR1, PHF8, KDM5C, SYNGAP1, DYRK1A, GRIN2B, IQSEC2, FOXG1, UBE3A, SYNGAP1, SLC9A6, TCF4, ZEB2) using Ampliseq approach developed by Life Technologies. All blood samples were obtained after receiving informed consent. This study was prospectively reviewed and approved by our local research ethics committee. Genomic DNA of the affected girl was amplified by Ampliseq approach, and subjected to sequencing on PGM Life Technologies sequencers (Platform at Jean Dausset, Institut Cochin, Paris, France). No pathogenic anomalies were identified by sequencing and CGH microarray. Second, taking into account these data, we performed whole-exome sequencing (WES).

\section{Whole-exome sequencing}

Library generation, exome enrichment, and WES were performed at the French National Centre for Genotyping (CNG, Evry, France). Briefly, libraries were prepared from $3 \mu \mathrm{g}$ genomic DNA extracted from whole blood using an optimized SureSelect Human Exome kit (Agilent, Santa Clara, CA, USA) following the manufacturer's instructions. Captured, purified, and clonally amplified libraries targeting the exome were then sequenced on a HiSeq 2000 (Illumina, San Diego, CA, USA) according to the manufacturer's recommendations. Obtained sequence reads were aligned to the human genome (hg19) using BWA software. Downstream processing was carried out with Genome analysis toolkit (GATK), SAMtools, and Picard Tools (http://picard.sourceforge. net/). ${ }^{14,15}$ Single-nucleotide variants and indels were subsequently called by SAMtools suite (mpileup, bcftools, vcfutil). All calls with a read coverage $\leq 5 \times$ and a Phred-scaled SNP quality of $\leq 20$ were filtered out. Substitution and variation calls were performed with SAMtools pipeline (mpileup). The variants were annotated with an in-house Paris Descartes bioinformatics platform pipeline based on the Ensembl database (release 67). ${ }^{16}$ An autosomal dominant approach was applied for candidate gene identification. After exome analysis, each selected variants were confirmed by polymerase chain reaction (PCR) using genomic DNA from the patients and their parents and direct sequencing using BigDyedideoxy terminator chemistry and an ABI3130xl genetic DNA analyzer (Applied Biosystems, Thermo Fisher Scientific, Waltham, MA, USA). Primer sequences and positions, PCR conditions, and product sizes are available on request.

RNA isolation, cDNA synthesis, and quantitative real-time PCR After informed consent was obtained from the parents, a skin biopsy was carried out for the female individual with intellectual disability. Total RNA from primary cultures of fibroblasts was converted to cDNA with Maxima First Strand cDNA synthesis Kit (ThermoScientific, Rochester, NY, USA) for quantitative real-time PCR with SYBR Green as detection agent with LightCycler480 detection system (Roche Applied Bioscience, Indianapolis, IN, USA). Primer sequences are described in Supplementary Table S1. After PCR amplification, a dissociation protocol was performed to determine the melting curve of PCR product. Reactions with melting curves indicating a single amplification product were considered positive for further analysis. The identity and expected size of the single PCR product were also confirmed by sequencing. For each cycle, a relative quantification of each individual gene amount was calculated by the comparative $\Delta \Delta \mathrm{CT}$ method as described by the manufacturer, providing a relative measure of expression for genes of interest, which were all normalized. Normalization factor was based on the mean of control gene $U B C$ (ubiquitin conjugating enzyme) selected because of its expression stability in human fibroblasts. ${ }^{17}$ Statistical analysis was carried out using nonparametric Mann-Whitney test (Prism, GraphPad software Inc., La Jolla, CA, USA). A $P$-value $<0.05$ was considered as significant.

\section{Description of sequence variants}

Sequence variants in $\mathrm{KCNCl}$ gene are numbered starting from the first base of the ATG codon, numbering based on reference sequence NM_004976.4. Description of the sequence (Human Genome Variation Society, http://www. hgvs.org/mutnomen/recs.html) was done with the assistance of Alamut Visual software version 2.4.2 (Interactive Biosoftware, Rouen, France). KCNC1 variant has been submitted to the specific gene variant database LOVD (http:// databases.lovd.nl/shared/variants/0000147175). The patient ID is 00089106 and the variant ID is 0000147175 . The other potential variants are 0000147174 , 0000147176-0000147181.

\section{RESULTS}

Exome analysis revealed a nonsense variant in the $\mathrm{KCNC1}$ gene The pedigree structure of this family was consistent with autosomal dominant inheritance. Clinical examination did not suggest a specific syndromic form of intellectual disability. Previous candidate gene investigations and CGH array had not identified the causative gene. We performed exome sequencing on three members from the family (father, mother, and affected daughter). Summary of exome 
Table 1 Summary of exome sequencing data for the proband (individual-3) and her parents (individuals 1 and 2)

\begin{tabular}{lrrc}
\hline & Father & Mother & Proband \\
\hline Total captured regions size $(\mathrm{Mb})$ & & & \\
$\quad$ Average depth for nucleotides in captured region & 180 & 178 & 129 \\
$\%$ Of captured regions with coverage $>15$ & 93 & 93 & 93 \\
\% Of captured regions with coverage $>30$ & 85 & 88 & 78 \\
& & & \\
Average coverage of captured region (\%) & & & \\
$\quad$ Total number of SNPs (essential splicing and & 16579 & 17640 & 15150 \\
coding regions) before filtering & & & \\
Total number of INDELs (essential splicing and & 814 & 875 & 772 \\
coding regions) before filtering & & & \\
& & & \\
Total number of SNPs & 522 & 545 & 225 \\
Total number of INDELs & 15 & 19 & 11 \\
$N$ rare homozygous & & & 5 \\
$N$ compound heterozygous & & & 1 \\
$N$ X linked & & & 13 \\
$N$ de novo events & & & 111 \\
\hline Inherited from her father & &
\end{tabular}

sequencing data for the proband and her parents is reported in Table 1. In total, 15150 to 17640 SNP (essential splicing and coding regions) and 772 to 875 small deletions/insertions (1-10 bp) (INDEL) were identified across the exomes. We tested an autosomal dominant inheritance and applied five consecutive filters to narrow down the variants: (i) variants heterozygous in the father and affected child and not present in the mother; (ii) variants with read depth $>10$; (iii) exclusion of $\mathrm{X}$ chromosome-linked variants; (iv) rare variants in the population $(\mathrm{MAF}<1 \%$ in the 1000 genomes, ExAC database, and dbSNP137); (v) variants with moderate high or functional impact (Polyphen-2 and SIFT scores). After filtering these data, it resulted in the identification of candidate variants in eight genes (KCNC1, TPH1, MADD, EGFR, CES5A, GADL1, SLC35A5, and KIAA1024; Table 2). Except one, all variants were missense variants. Segregation analysis by using affected (individual-4) and unaffected (individual-5) members of this family allowed us to exclude four variants (c.2842G $>$ A (p.V948I) in EGFR; c.971T > C (p.L324P) in SLC35A5, c.211G > A (p.V71M) in GADL1, and c.473A $>$ G (p.D158G) in CES5A). Four variants were not excluded (c.689G $>$ A (p.R230H) in TPH1; c.1018G $>$ A (p.V340M) in MADD, c.1015C $>\mathrm{T}\left(\mathrm{p} . \mathrm{R} 339^{*}\right)$ in KCNC1, c.1832A $>\mathrm{T} \quad(\mathrm{p} . \mathrm{E} 611 \mathrm{~V})$ in KIAA1024). Taking into account that KCNC1 was previously involved in progressive myoclonus epilepsies, nonsense variant (c.1015C $>\mathrm{T}$ ( $\mathrm{p}$. $\left.\left(\mathrm{R} 339^{*}\right)\right)$ ) in the $\mathrm{KCNC1}$ gene was identified as the most plausible candidate. ${ }^{13} \mathrm{KCNC1}$ variant was confirmed by Sanger sequencing for both affected siblings (boy and daughter) and affected father (Figure 1a). Moreover, both unaffected individuals (mother and unaffected girl) do not carry this nonsense variant.

Relative quantification of $K C N C 1$ transcript showed that mutated transcript was target for the nonsense-mediated RNA decay Despite an expected tendency for premature termination codons to yield shortened polypeptide products, formation of truncated proteins does not occur often in vivo. Many organisms, including humans, use a nonsense-mediated mRNA decay pathway, which degrades mRNAs containing nonsense variants before translation into nonfunctional polypeptides. To understand molecular consequences of this nonsense variant, we investigated mRNA processing by detection of its transcript in fibroblasts obtained from a skin biopsy of one of the affected patients. Quantification of mRNA showed a very significant reduction ( $>50 \%$ ) of $\mathrm{KCNC1}$ transcript in fibroblasts from the affected patient compared with 13 independent controls (Figure 1b). This result suggests that the mutant transcript is highly unstable leading to an absence of truncated protein synthesis and reduction of $\mathrm{KCNC1}$ expression.

\section{DISCUSSION}

Using whole-exome sequencing, we identified eight candidate variants in this family with ID. Segregation analysis by comparing other unaffected and affected individuals from this family, allowed us to exclude four missense variants. Three of them (c.689G > A, p. (R230H) in TPH1; c.2842G > A p.(V948I) in EGFR; c.971T >C p.(L324P) in SLC35A5) were also previously described in the ExAC database (http:// exac.broadinstitute.org/). We cannot exclude the TPH1, MADD, and KIAA1024 variants by segregation analysis. However, Tryptophan hydroxylase-1 (TPH1) is only expressed by non-neuronal cells including enterochromaffin cells of gut, mast cells, and pineal gland, and it has not been found to have any differences between the Tph1deficient and wild-type mice in general motor behavior as tested by rotarod, grip-strength test, open field, and beam walk. ${ }^{18}$ KIAA1024 located in 15q25.1 presents more than 350 missense variants in ExAC database including several in the same region (p.(R608Q), p.(S614L), p.(R617C)). Finally, MADD, a splice variant of IG20 gene, encodes a protein highly expressed in melanocytes, parotid acinar cells, and involved in physiological cell death. ${ }^{19-21}$ Taking into account of all these data, we focused on the KCNC1 gene previously involved in myoclonus epilepsies. ${ }^{13} \mathrm{Up}$ to now, only one loss-of-function variant was described in ExAC database (c.1504+1G > T, 1/117116). Patients described here are the first cases presenting a loss-of-function variant of $\mathrm{KCNC1}$ gene in intellectual disability context, suggesting that KCNC1 variants can also cause ID.

KCNC1 encodes $\mathrm{K}_{\mathrm{V}} 3.1$, which functions as a highly conserved potassium ion channel subunit of the $\mathrm{K}_{\mathrm{V}} 3$ subfamily of voltage-gated tetrameric potassium ion channels. $\mathrm{K}_{\mathrm{V}} 3$ channel subunits consist of six membrane-spanning segments (S1-S6), have overlapping expression patterns, and can form heterotetramers. ${ }^{22}$ S4 constitutes the main voltage sensor where specific positively charged arginine residues contribute to the gating charge. ${ }^{23}$

Here, we showed that the mutated mRNA is degraded by nonsensemediated mRNA decay pathway, suggesting that it is unlikely translated into a nonfunctional polypeptide that would alter all $\mathrm{K}_{\mathrm{v}}$ 3-mediated currents of the neurons in which it is expressed. Most likely reduction of $\mathrm{KCNC1}$ protein expression (haploinsufficiency) is at the origin of the pathology.

This result explains that reduction of $\mathrm{KCNC1}$ expression due to nonsense variant is associated with ID without PME, whereas the p. $(\mathrm{R} 320 \mathrm{H})$ substitution associated with mutant protein synthesis causes a dominant-negative effect on wild-type $\mathrm{K}_{\mathrm{V}} 3.1$ channels and progressive myoclonus epilepsies. ${ }^{13}$

Interestingly, $\mathrm{K}_{\mathrm{v}} 3.1$ homozygous mice are viable and fertile indicating that functional $\mathrm{K}_{\mathrm{v}} 3.1 \mathrm{~K}^{+}$channels are neither essential for embryonic development nor for reproduction. In our family, the father bearing the nonsense mutation is also fertile. $\mathrm{K}_{\mathrm{v}} 3.1$ homozygous mice show normal spontaneous locomotion, unaltered acquisition (learning), and retention (memory) of a conditioned response, and no spontaneous seizures. However, they are smaller in body size and have impaired coordinated motor skills. ${ }^{24}$ As observed in $\mathrm{K}_{\mathrm{v}} 3.1$ homozygous mice, our affected patients have relatively mild phenotypes without seizures. 


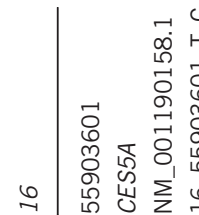

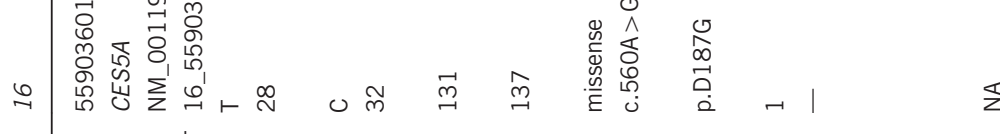

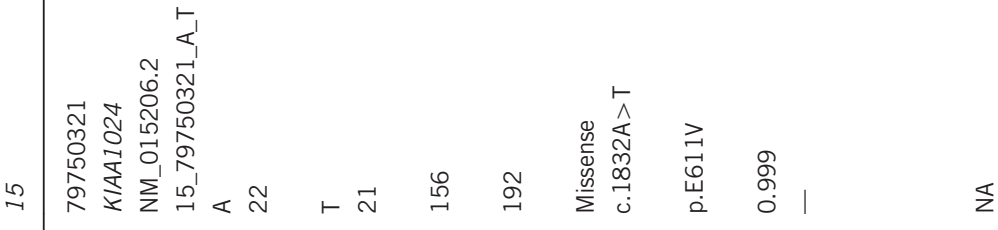

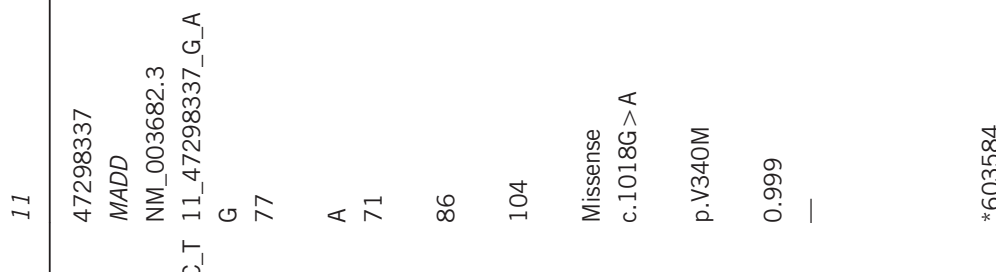

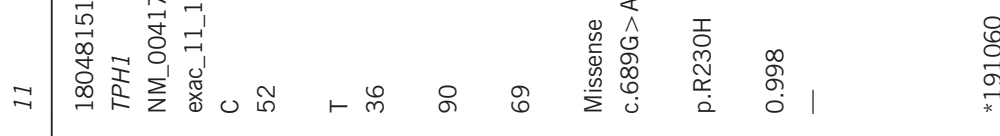

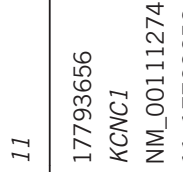

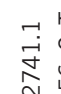

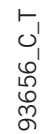

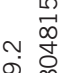

용

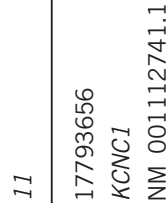

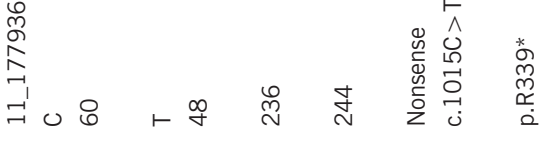

i

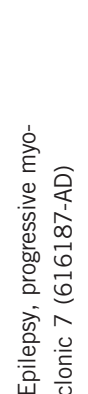

$\sqrt{1}$

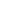

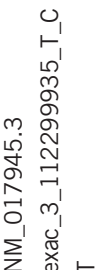

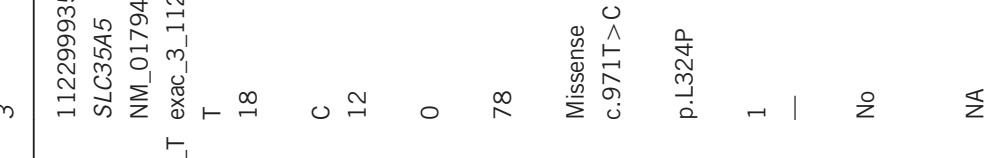

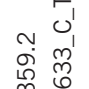

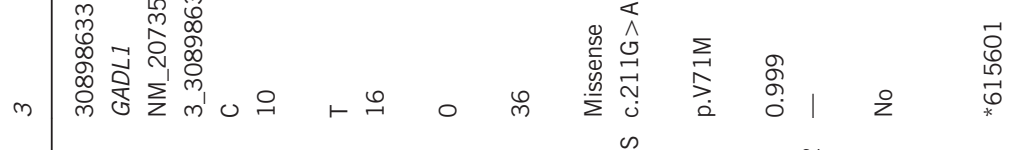

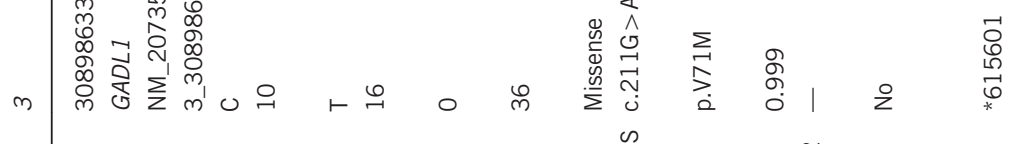

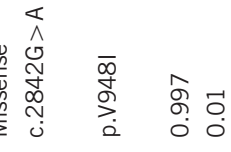

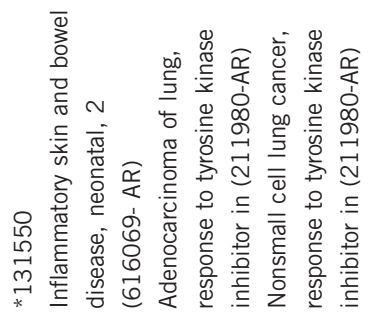

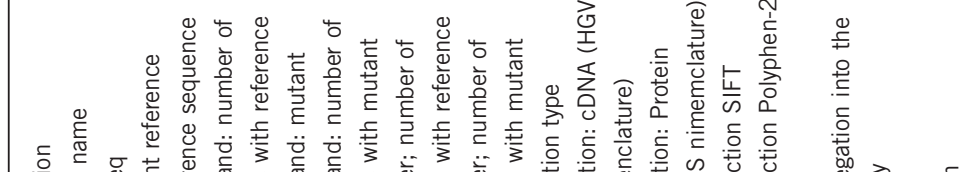

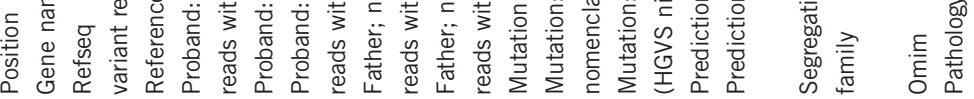


Early expression of $\mathrm{K}_{\mathrm{v}} 3$ channels during an embryonic period of life in which the fast firing phenotype is not yet present suggests that $\mathrm{K}_{\mathrm{v}} 3$ channels have other non-conventional functions. $\mathrm{K}_{\mathrm{v}} 3$ channels have not only been involved in cell proliferation, migration, and neuronal growth cone, but may also have a role in regulating neuronal and glial properties during the earliest phases of brain development. ${ }^{25-27}$ Our data suggest that $\mathrm{KCNC1}$ haploinsufficiency may affect at least one of these functions and could have a role in the cognitive symptoms of our ID individuals. Further studies are needed to evaluate the impact of KCNC1 loss-of-function variants on gating properties of $\mathrm{K}_{\mathrm{v}} 3.1$ channels. Modulation of $\mathrm{K}_{\mathrm{V}} 3$ channel function may provide a possibility for pharmacological intervention in patients with $\mathrm{KCNC1}$ variants. However, although anticonvulsant drugs targeting other $\mathrm{K}_{\mathrm{V}}$ channels exist, there is no activator of $\mathrm{K}_{\mathrm{V}} 3$ channels currently available. Nevertheless, taking into account the fact that $\mathrm{K}_{\mathrm{v}} 3.1$ channels are reduced in untreated schizophrenia and its expression is normalized with antipsychotics drugs, a similar treatment should be a future pharmacological approach in these KCNC1-related disorders. ${ }^{28}$

\section{CONFLICT OF INTEREST}

The authors declare no conflict of interest.

\section{ACKNOWLEDGEMENTS}

We thank the patients and their family for their participation in this study. We thank Aurélie Toussaint for her help to draft the manuscript. This work was supported by Fondation Maladies Rares. We thank the cell bank of Cochin Hospital (Patrick Nusbaum) for assistance with the samples used in this study.

1 Coetzee WA, Amarillo Y, Chiu J et al: Molecular diversity of K+ channels. Ann N Y Acad Sci 1999; 868: 233-285.

2 Jan LY, Jan YN: Voltage-gated potassium channels and the diversity ofelectrical signalling. J Physiol 2012; 590: 2591-2599.

3 Rudy B, McBain CJ: Kv3 channels. voltage-gated $\mathrm{K}+$ channels designed for highfrequency repetitive firing. Trends Neurosci 2001; 24: 517-526.

4 Vega-Saenz de Miera EC, Lin JW: Cloning of ion channel gene families using the polymerase chain reaction. Methods Enzymol 1992; 207: 613-619.

5 Lesage F, Guillemare E, Fink M et al: Molecular properties of neuronal G-proteinactivated inwardly rectifying K+ channels. J Biol Chem 1995; 270: 28660-28667.

6 Ozaita A, Martone ME, Ellisman MH, Rudy B: Differential subcellular localization of the two alternatively spliced isoforms of the Kv3.1 potassium channel subunit in brain. J Neurophysiol 2002; 88: 394-408.
7 Chang SY, Zagha E, Kwon ES et al: Distribution of Kv3.3 potassium channel subunits in distinct neuronal populations of mouse brain. J Comp Neurol 2007; 502: 953-972.

8 Deutsch C, Chen LQ: Heterologous expression of specific $\mathrm{K}+$ channels in T lymphocytes: functional consequences for volume regulation. Proc Natl Acad Sci USA 1993; 90: 10036-100340.

9 Tiwari-Woodruff S, Beltran-Parrazal L, Charles A, Keck T, Vu T: Bronstein J. K+ channel KV3.1 associates with OSP/claudin-11 and regulates oligodendrocyte development. Am J Physiol Cell Physiol 2006; 291: C687-C698.

10 Schaarschmidt G, Wegner F, Schwarz SC, Schmidt H, Schwarz J: Characterization of voltage-gated potassium channels in human neural progenitor cells. PLoS One 2009; 4: e6168.

11 Figueroa KP, Waters MF, Garibyan V et al: Frequency of KCNC3 DNA variants as causes of spinocerebellar ataxia 13 (SCA13). PLoS One 2011; 6: e17811.

12 Rajakulendran S, Roberts J, Koltzenburg M, Hanna MG, Stewart H: Deletion of chromosome 12q21 affecting KCNC2 and ATXN7L3B in a family with neurodevelopmental delay and ataxia. J Neurol Neurosurg Psychiatry 2013; 84: 1255-1257.

13 Muona M, Berkovic SF, Dibbens LM et al: A recurrent de novo mutation in KCNC1 causesprogressive myoclonus epilepsy. Nat Genet 2015; 47: 39-46.

14 DePristo MA, Banks E, Poplin R et al: A framework for variation discovery and genotyping using next-generation DNA sequencing data. Nat Genet 2011; 43: 491-498.

15 Li H, Handsaker B, Wysoker A et al: 1000 Genome Project Data Processing Subgroup: the sequence alignment/map format and SAMtools. Bioinformatics 2009; 25: 2078-2079.

16 Flicek P, Ahmed I, Amode MR et al: Ensembl 2013. Nucleic Acids Res 2013; 41 : D48-D55.

17 Lanzafame M, Botta E, Teson M et al: Reference genes for gene expression analysis in proliferating and differentiating human keratinocytes. Exp Dermatol 2015: 24: 314-316.

18 Suidan GL, Duerschmied D, Dillon GM et al: Lack of tryptophan hydroxylase-1 in mice results in gait abnormalities. PLoS One 2013; 8: e59032.

19 Miyoshi J, Takai Y: Dual role of DENN/MADD (Rab3GEP) in neurotransmission and neuroprotection. Trends Mol Med 2004; 10: 476-480.

20 Turner A, Li LC, Pilli T et al: MADD knock-down enhances doxorubicin and TRAIL induced apoptosis in breast cancer cells. PLoS One 2013; 8: e56817.

21 Imai $A$, Ishida M, Fukuda M, Nashida T, Shimomura H: MADD/DENN/Rab3GEP functions as a guanine nucleotide exchange factor for Rab27 during granule exocytosis of rat parotid acinar cells. Arch Biochem Biophys 2013; 536: 31-37.

22 Seoh S-A, Sigg D, Papazian DM, Bezanilla F: Voltage-sensing residues in the S2 and S4 segments of the shaker K+ channel. Neuron 1996; 16: 1159-1167.

23 Aggarwal SK, MacKinnon R: Contribution of the S4 segment to gating charge in the shaker K+ channel. Neuron 1996; 16: 1169-1177.

24 Ho CS, Grange RW, Joho RH: Pleiotropic effects of a disrupted K+ channel gene: reduced body weight, impaired motor skill and muscle contraction, but no seizures. Proc Natl Acad Sci USA 1997; 94: 1533-1538.

25 Boda E, Hoxha E, Pini A, Montarolo F, Tempia F: Brain expression of Kv3 subunits during development, adulthood and aging and in a murine model of Alzheimer's disease. J Mol Neurosci 2012; 46: 606-615.

26 Pollock NS, Ferguson SC, McFarlane S: Expression of voltage-dependent potassium channels in the developing visual system of Xenopus laevis. J Comp Neurol 2002; 452: 381-391.

27 Liebau S, Pröpper C, Böckers T et al: Selective blockage of Kv1.3 and Kv3.1 channels increases neural progenitor cell proliferation. J Neurochem 2006; 99: 426-437.

28 Yanagi M, Joho RH, Southcott SA, Shukla AA, Ghose S, Tamminga CA: Kv3.1containing $\mathrm{K}(+)$ channels are reduced in untreated schizophrenia and normalized with antipsychotic drugs. Mol Psychiatry 2014; 19: 573-579.

Supplementary Information accompanies this paper on European Journal of Human Genetics website (http://www.nature.com/ejhg) 